\title{
Clinical Characteristics of a Group of Cameroonian Neonates with Delayed Breastfeeding Initiation
}

\author{
Georges Pius Kamsu Moyo*, Ngwanou Dany Hermann \\ Faculty of Medicine and Biomedical Sciences, University of Yaounde 1, Yaounde, Cameroon
}

Email address:

kamsuzicfried@yahoo.fr (G. P. K. Moyo)

${ }^{*}$ Corresponding author

\section{To cite this article:}

Georges Pius Kamsu Moyo, Ngwanou Dany Hermann. Clinical Characteristics of a Group of Cameroonian Neonates with Delayed Breastfeeding Initiation. American Journal of Pediatrics. Vol. 6, No. 3, 2020, pp. 285-288. doi: 10.11648/j.ajp.20200603.28

Received: June 22, 2020; Accepted: July 4, 2020; Published: July 17, 2020

\begin{abstract}
The timely or Early Initiation of Breastfeeding (EIBF) within the first hour following childbirth is known to be a determining factor for the overall breastfeeding process. Nevertheless, in some cases there may exist a considerable delay in its start. This could be responsible for disorders in neonate infants, with varying severity. The main objective of this survey was to determine particular clinical characteristics of a group of neonates with delayed breastfeeding initiation, compared with that of neonates with early breastfeeding initiation (EBFI), during the first week of life. We did an analytical cross-sectional study with the notion of timely breastfeeding initiation according to the World Health Organization (WHO), used to distinguish neonates. The study was conducted at the Yaoundé Gynaeco-Obstetric and Paediatric Hospital from December 2018 to May 2019. All livebirth infants weighing $>2000 \mathrm{~g}$ were recruited during the period of study. From an overall sample of 250 neonates, $153(61.2 \%)$ had delayed breastfeeding initiation, while 97 others had EIBF. All neonates with delayed breastfeeding initiation had other characteristics of inadequate breastfeeding practices. Although 144 (94\%) were in good health immediately after birth, $38(24.8 \%)$ were hospitalized within 7 days, of which $21(55.2 \%)$ were related to sepsis and $6(15.7 \%)$ due to metabolic disorders. They represented $83.3 \%$ (15 out of 18) infants with hypotrophy. Therefore, the late initiation of breastfeeding may induce inadequate breastfeeding practices, with significant repercussions on the neonate's health condition, including nutritional disorders such as metabolic disturbances, growth retardation, increased susceptibility to neonatal infections and eventually, the rate of hospitalization during the given period of time on. Mothers' education, medical staff and family encouragements may help promote timely breastfeeding initiation, so as to limit adverse neonatal outcomes.
\end{abstract}

Keywords: Early Breastfeeding, Breastfeeding Initiation, Newborn, Yaoundé

\section{Introduction}

The Early initiation of breast feeding is also known as timely breastfeeding and may be defined as the percentage of newly born infant who are breastfed within the first hour following delivery [1]. In effect, it is believed that the first breastmilk produced or colostrum that is secreted few minutes after delivery is the richest, considered as the first vaccine due to its valuable immunological content. Moreover, its consistence in terms of high quality nutrients makes it fundamental for the neonate's protection and immediate dietary needs [1]. Timely breastfeeding prevents hypoglycemia and hypothermia, as it serves as fuel for the Kreb's cycle which is indispensable for energy production.
There is enough evidence that breastfeeding decreases neonatal mortality, morbidity, sepsis-related deaths, diarrhea and respiratory infections in neonates and children $[2,3]$. As a matter of fact, a number of research studies advocate the fact that breastfeeding largely contributed to the reduction of about 804.000 deaths (11.6\%) among under-five children worldwide in 2011 [4, 5]. However, some undesired events such as caesarean delivery or "initial agalactorrhea" in several women may be responsible for considerable delays in breastfeeding initiation and eventually the whole breastfeeding process [6]. This may be responsible for numerous disorders in neonates, including nutritional and metabolic anomalies, increased rates of hospitalization for sepsis or other pathologies, as well as growth and developmental disorders on a long coarse [2, 3]. 
The present study aimed at describing, based on analyses, the various clinical conditions that may be associated with delayed breastfeeding initiation. Indeed, although some studies have shown protective effects of early breastfeeding initiation against obesity and other chronic diseases on the long-term, few of them give account of potential neonatal disorders due to delayed breastfeeding initiation [2, 3]. Nevertheless, countries such as India have made it mandatory to keep newly delivered mothers and babies in the hospital for at least 48 hours in case of normal delivery and seven days in case of caesarean section so as to encourage breastfeeding under the watchful eyes of the medical staff [7]. This had a significant impact on the enhancement of breastfeeding practices and the prevention of adverse neonatal outcomes.

\section{Methodology}

This was an analytical cross-sectional study which was carried out over a period of six months, from December 2018 to May 2019. The survey took place at the Yaoundé Gynaeco-Obstetric and Paediatric Hospital which is a University Teaching Hospital in Cameroon. We included 7 days old neonate infants delivered at the said hospital with birthweights $>2000 \mathrm{~g}$ and without contraindication to breastfeeding, nor obvious congenital digestive malformations, susceptible to alter the breastfeeding process. Mothers were provided with necessary explanations and information sheets about the survey, on the day of the routine 1-week postnatal visit. Written and verbal consents were then obtained, pregnancy, perinatal, and immediate neonatal histories were retrieved from medical records and patients' files. Emphasis was laid on adequate breastfeeding practices including timely initiation, exclusiveness, effectiveness and duration according to the World Health Organization's (WHO) recommendations. Neonates were then wholly examined and checked for anthropometric parameters, to enable comparative analyses in terms of hospitalization rate, infection, jaundice, nutritional and metabolic disorders, growth rate, and mortality rate. Sampling was consecutive and exhaustive during the study period. Useful information was recorded and analyzed using CS Pro version 6.2 and SPSS version 20.0. Chi-square testing was used to establish statistical associations between the variables. The $\mathrm{P}$ value $(\mathrm{p})<0.05$ was used to characterize any statistically significant results. While the Odds Ratio (OR) with its $95 \%$ Confidence Interval (CI) was used to establish the risk relationships.

The various operational terms used were early breastfeeding, defined here as the initiation of the process within the first hour following delivery. Breastfeeding assessment based on measurable criteria such as the child's vigor in suckling, the type of arousal and the presence of swallowing were used for qualifying the act as successful or not.

Ethical clearances from the Institutional Ethics and Research Committee of the Faculty of Medicine and Biomedical sciences of the University of Yaoundé 1 and the Yaoundé Gynaeco-Obstetric and Paediatric Hospital were obtained before the beginning of the survey. The data collected was kept strictly confidential and used only for the purposes of the study.

\section{Results}

The birth process was eutocic in 102 cases (66.6\%), and vaginal route was the main mode of delivery in 105 women $(68.6 \%)$. The newborns had a mean gestational age of $37.8 \pm$ 1.6 weeks and a mean birth weight of $3038.7 \pm 400.3 \mathrm{~g}$. The male sex predominated by a ratio of 1.3 (86 males, 66 females).

\subsection{Delayed Breastfeeding Initiation}

The average time to start breastfeeding after delivery in our series was 120 minutes ( 2 hours). Only 97 (38.8\%) mothers had put the baby on to the breast within the first hour following delivery (Table 2).

Table 1. Distribution according to breastfeeding initiation.

\begin{tabular}{lll}
\hline Variables & N & Percentage (\%) \\
\cline { 1 - 2 } Time of breastfeeding initiation (minutes) & & \\
\hline$>60$ & 153 & 61.2 \\
$\leq 60$ & 97 & 38.8 \\
$<30$ & 49 & 19.6 \\
{$[30-60]$} & 48 & 19.2 \\
\hline
\end{tabular}

\subsection{Inadequate Breastfeeding Practices}

This classification was based on four criteria, including timely initiation of the process, effectiveness, exclusiveness and duration, according to WHO recommendations.

Table 2. Characteristics of inadequate breastfeeding.

\begin{tabular}{lll}
\hline Variables & N (250) & Percentage (\%) \\
\hline $\begin{array}{lll}\text { Inadequate breastfeeding practices } \\
\text { Delayed breastfeeding initiation }>60\end{array}$ & 153 & 61.2 \\
$\begin{array}{l}\text { Minutes } \\
\text { Formula use or other breastmilk }\end{array}$ & 153 & 61.2 \\
$\begin{array}{l}\text { Substitutes } \\
\text { Ineffective breastfeeding technique }\end{array}$ & 102 & 66.8 \\
$\begin{array}{l}\text { No intention for 6 months exclusive } \\
\text { Breastfeeding }\end{array}$ & 51 & 50.3 \\
\hline
\end{tabular}

\subsection{Clinical Characteristics of Neonates}

The various clinical characteristics of neonates are summarized in table 3 . 
Table 3. Clinical Characteristics of neonates.

\begin{tabular}{|c|c|c|c|c|}
\hline \multirow[b]{2}{*}{ Variables } & \multicolumn{2}{|c|}{ Timely breastfeeding initiation } & \multirow{2}{*}{ OR (IC à 95\%) } & \multirow{2}{*}{ p-value } \\
\hline & $>60 \min$ (cases) & $\leq 60 \min ($ controls $)$ & & \\
\hline \multicolumn{5}{|l|}{ Normal state at birth } \\
\hline Yes & $144(60)$ & $96(40)$ & $0.2(0.02-1.3)$ & 0.094 \\
\hline No & $9(90)$ & $1(10)$ & & \\
\hline \multicolumn{5}{|c|}{ Hospitalization in the mean time } \\
\hline Yes & $38(63.3)$ & $22(36.7)$ & $1.1(0.6-2.1)$ & 0.697 \\
\hline No & $115(60.5)$ & $75(39.5)$ & & \\
\hline \multicolumn{5}{|l|}{ Pathology } \\
\hline Neonatal sepsis & $13(92.9)$ & $1(7.1)$ & $10.9(1.3-90.5)$ & 0.009 \\
\hline Birth Asphyxia & $3(75)$ & $1(25)$ & $1.8(0.2-18.4)$ & 1.000 \\
\hline Jaundice & $857.1)$ & $6(42.9)$ & $0.7(0.2-2.4)$ & 0.583 \\
\hline Risk of sepsis & $8(53.3)$ & $7(46.7)$ & $0.6(0.2-1.1)$ & 0.353 \\
\hline Métabolic disorders & $6(46.2)$ & $7(53.8)$ & $0.4(0.1-1.4)$ & 0.146 \\
\hline \multicolumn{5}{|l|}{ Weeks of gestation } \\
\hline$<37$ & $18(85.7)$ & $3(14.3)$ & $4.2(1.2-14.6)$ & 0.016 \\
\hline$[37-42]$ & $130(58.6)$ & $92(41.4)$ & $0.3(0.1-0.8)$ & 0.016 \\
\hline$\geq 42$ & $5(71.4)$ & $2(28.6)$ & $1.6(0.3-8.4)$ & 0.709 \\
\hline \multicolumn{5}{|l|}{ Weight at 7 days } \\
\hline$<2500$ & $15(83.3)$ & $3(16.7)$ & $3.4(1.01-12.1)$ & 0.045 \\
\hline$[2500-4000]$ & $128(59.3)$ & $88(40.7)$ & $0.5(0.2-1.2)$ & 0.112 \\
\hline$\geq 4000$ & $10(62.5)$ & $6(37.5)$ & $1.1(0.4-3.01)$ & 0.912 \\
\hline
\end{tabular}

\section{Discussion}

The rate of delayed Breastfeeding initiation in this survey was $61.2 \%$, which is quite higher than values obtained in most developed countries where the culture of breastfeeding is supposedly lower. This is the case of countries such as Australia, where the percentage of mothers, and hence neonates with timely breastfeeding initiation among some communities could be as high as $98 \%$ [8]. Our results are also higher than incidences reported in some Middle-East countries such as Saudi Arabia (22.2\%), Nepal (33.6\%), as well as in some African developing countries such as Ethiopia $(26.9 \%)$, where relatively lower percentages of mothers and neonates with delayed breastfeeding initiation have been reported [9-12]. Possible reasons to this discrepancy may be a low level of education and a weakness of antenatal counselling, as far as the necessity to promptly initiate breastfeeding is concerned. More so, increasing women job occupation, adopted cultures of breastmilk substitution with formula, and the hyper "sexualization" of the breast to the detriment of its lactation function may have this adverse effect of reducing adequate breastfeeding practices among African women [7, 9, 13-18]. The end result of these new habits in African women, could be responsible for reduced neonatal protection in terms of food security and immunity. Furthermore, it appeared that all neonates with delayed breastfeeding initiation had other characteristics of inadequate breastfeeding as well, including ineffectiveness of the act, unexclusiveness, and briefness.

In this survey, we chose to reassess neonates after 1 week of life and not later on because of three main reasons. Firstly, for convenience reasons, due to the fact that routine assessment of neonates at 1 week is systematic at the Yaoundé Gynaeco-Obstetric and paediatric hospital, just as in several other referral hospitals in Cameroon. Secondly, this period corresponds to the time when postnatal counselling is most reinforced in order to promote breastfeeding practice and immunization, including the cross-checking of immunization status and vaccines. As such, BCG and Oral Polio vaccines which are ideally administered as from the first day or week after birth according to the current Enlarged Program for Immunization (EPI) in Cameroon could be controlled. The third reason is that during this period, developing disorders may be promptly dealt with. Given that some hypothesis suggest neonatal emergencies mostly occur within the first week of life, described as the most critical period for neonate infants [19-22].

In fact, the overall rate of hospitalization within the first week among neonates enrolled in this survey was $24 \%$, out of which more than $63 \%$ were neonates with delayed breastfeeding initiation. The mortality rate in this group was $7.9 \%$ (3 out of 38 neonates), while that in the group of neonates with early breastfeeding initiation was lower at $4.5 \%$ ( 1 out of 22 neonates). From this, it may be suggested that neonates with delayed breastfeeding initiation had weaker immunological protection and were therefore likely to develop illnesses which might have been fatal in some cases.

In effect, a statistically significant association occurred between the onset of neonatal sepsis and the notion of delayed breastfeeding initiation at birth, with close to $55 \%$ of hospitalizations in such infants being related to sepsis. Moreover, $85.7 \%$ preterm infants enrolled in this survey were not breastfed in due time and required adapted feeding to catch-up with their growth process. Whereas, $83.3 \%$ neonates with hypotrophy within this time interval, were those whom had a delayed breastfeeding initiation. These results seem coherent, as contrarily to adequate breastfeeding, failure to early initiate the breastfeeding process may pave the way for other feeding malpractices in neonates, responsible for increased rates of morbidity and mortality in high risk 
infants, including those with infection, prematurity, and low birth weight [19-22].

\section{Conclusion}

Delayed breastfeeding initiation may lead to other breastfeeding malpractices such as ineffectiveness of the act, unexclusiveness, and briefness which may contributing to the failure of the process. This may have considerable repercussions on neonates' health conditions, leading to increased rates of nutritional disorders such as growth retardation, metabolic disturbances and infectious disorders, with a direct effect on the rate of hospitalization among this population. Nevertheless, mothers' education, medical staff and family encouragements may help to promote timely breastfeeding initiation, so as to limit adverse neonatal outcomes.

\section{Funding}

Private.

\section{Conflict of Interest}

The authors declare that they have no competing interest.

\section{Acknowledgements}

Hospitals authorities, all collaborators to this project.

\section{References}

[1] WHO. http://www.who.int/topics/breastfeeding/en/.2020.

Breastfeeding.

[2] Hess C, Ofei A, Mincher A. Breastfeeding and childhood obesity among African americans: a systematic review. MCN Am J Matern Child Nurs. 2015; 40 (5): 313-9.

[3] Khan J, Vesel L, Bahl R, Martines J. Timing of breastfeeding initiation and exclusivity of breastfeeding during the first month of life: effects on neonatal mortality and morbidity--a systematic review and meta-analysis. Matern Child Health J. 2015; 19 (3): 468-79.

[4] Black R, Victora C, Walker S, Bhutta Z, Christian P, de, Onis $\mathrm{M}$, et al. Maternal and child undernutrition and overweight in low-income and middle-income countries. Lancet. 2013; 382: 427-51.

[5] Quyen Thi-Tu B, Hwa-Young L, Thi-Kim L, DoVan D, Lan ThiHoang V. Trends and determinants for early initiation of and exclusive breastfeeding under six months in Vietnam: results from the Multiple Indicator Cluster Surveys, 2000 2011. Global Health Action. 2016; 9 (1): 29433.

[6] Kuyper E, Vitta B, Dewey K. Implications of cesarean delivery for breastfeeding outcomes and strategies to support breastfeeding. Alive Thrive Tech Brief. 2014; 8: 1-9.

[7] Setegn T, Gerbaba M, Belachew T. Determinants of timely initiation of breastfeeding among mothers in Goba Woreda,
South East Ethiopia: A cross sectional study. BMC Public Health. 2011; 11: 217.

[8] Kimani-Murage E, Kyobutungi C, Ezeh A, Wekesah F, Wanjohi M, Muriuki P, et al. Effectiveness of personalized, home-based nutritional counselling on infant feeding practices, morbidity and nutritional outcomes among infants in infants in Nairobi slums: study protocol for a cluster randomised controlled trial. Trials. 2013; 14: 445.

[9] Adugna D. Women's perception and risk factors for delayed initiation of breastfeeding in Arba Minch Zuria, Southern Ethiopia. Int Breastfeed J. 2014; 9 (1): 8.

[10] MaJra J, ViJay K. Barriers to Early Initiation and Continuation of Breastfeeding in a Tertiary care Institute of Haryana: A Qualitative Study in Nursing Care Providers. J of Clin and Diagn Res. 2016 Sep; 10 (9): 16-20.

[11] Federal ministry of health. Health sector development program IV Woreda based annual core plan. Australia; 2010. Report No.: 2.

[12] Bimerew A, Teshome M, Kassa G. Prevalence of timely breastfeeding initiation and associated factors in Dembecha district, North West Ethiopia: a cross-sectional study. Int Breastfeed J. 2016; 11: 28.

[13] Wolde T, Birhanu T, Ejeta I. Prevalence and determinants of timely initiation of breastfeeding among lactating mothers of urban dwellers in western Ethiopia. Food Sci Qual Manag. 2014; 31 (110): 2225-557.

[14] McLachlan H, Forster D. Initial breastfeeding attitudes and practices of women born in Turkey, Vietnam and Australia after giving birth in Australia. Int Breastfeed J. 2016; 1: 7.

[15] Amin T, Hablasa H, Qader A. Determinants of initiation and exclusivity of breastfeeding in Al Hassa, Saudi Arabia. Int Nurs Rev. 2010; 6 (2): 59-68.

[16] Girard L, Cote S, de Lauzon-Guillain B, Dubois L, Falissard B, Forhan A. Factors Associated with Breastfeeding Initiation: A Comparison between France and French-Speaking Canada. PLoS ONE. 2016; 11 (11): 1-14.

[17] Kelishadi R, Rashidian A, Jari M, Khosravi A, Khabiri R, Elahi E. A national survey on the pattern of breastfeeding in Iranian infants: The IrMIDHS study. Med J Islam Repub Iran. 2016 Oct; 30: 425.

[18] Sharma K, Byrne A. Early initiation of breastfeeding: a systematic literature review of factors and barriers in South Asia. Int Breast J. 2016; 11: 17.

[19] Georges Pius Kamsu Moyo, Donleine Sobguemezing, Hélène Tetinou Adjifack. Neonatal Emergencies in Full-term Infants: A Seasonal Description in a Pediatric Referral Hospital of Yaoundé, Cameroon. American Journal of Pediatrics. Vol. 6, No. 2, 2020, pp. 87-90. doi: 10.11648/j.ajp.20200602.13.

[20] Barousseau T, Sharieff G. Newborn emergencies: the first 30 days of life. Pediatr Clin North Am. 2006; 53 (1): 69-84.

[21] Pineda R. Breastfeeding practices in the neonatal intensive care unit before and after an intervention plan. Gainesville: University of Florida; 2006 p. 99.

[22] World Health Organization. Global strategy for infant and young child feeding. Geneva: World Health Organization; 2003. Report No.: 14. 